Letters to the Editor

\title{
Optical Resolution of Thiol Enantiomers by High-Performance Liquid Chromatography Using Precolumn Fluorescence Derivatization with o-Phthalaldehyde and $\alpha$-Amino Acids
}

\author{
Akira Sano, Shoji Takitani and Hiroshi Nakamura ${ }^{\dagger}$ \\ Faculty of Pharmaceutical Sciences, Science University of Tokyo, \\ Ichigaya-funagawara-machi, Shinjuku, Tokyo 162, Japan
}

Keywords Optical resolution, thiol enantiomer, chiral derivatization, $o$-phthalaldehyde, high-performance liquid chromatography, $\alpha$-amino acid

Various reagents for chiral derivatization have been developed for the resolution of biologically and pharmaceutically important enantiomers by high-performance liquid chromatography (HPLC).,2 However, regarding the chiral derivatization of thiols, only one chromogenic reagent, $2,3,4,6$-tetra- $O$-acetyl- $\beta$-D-glucopyranosyl isothiocyanate (GITC) ${ }^{3}$, has been reported so far. In order to improve the detection sensitivity, we therefore searched for a fluorogenic reaction suitable for the chiral derivatization of thiols. It is well known that the<smiles>O=Cc1ccccc1C=O</smiles><smiles>CC(C)(C)[C@H](N)C(=O)O</smiles>
L-Val D, L-Cys
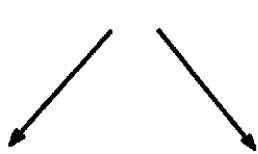<smiles>CC(C)[C@@H](CSc1c2ccccc2cn1[C@@H](CN)C(=O)O)[C@H](C)C(=O)O</smiles>

D-Cys derivative

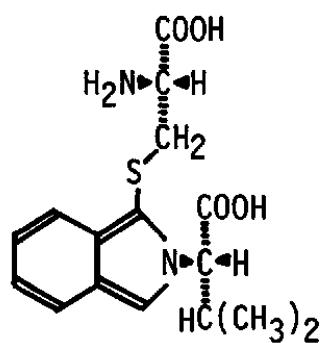

L-Cys derivative
Fig. 1 Derivatization of D,L-Cys with OPA and L-valine.

† To whom correspondence should be addressed. enantiomers of primary amines are readily resolved on a reversed-phase column when derivatized with $o$ phthalaldehyde (OPA) and a chiral thiol, such as Boc-Lcysteine $^{4}$ and $N$-acetyl-L-cysteine ${ }^{5}$, to form diastereomeric isoindole derivatives. The OPA fluorogenic reaction is also applicable to thiols. ${ }^{6,7}$ Based on these findings, we have devised an HPLC method for the simultaneous separation of enantiomeric thiols, such as cysteine (Cys) and homocysteine (HomoCys), using OPA and L-amino acids as chiral derivatization reagents (Fig. 1).

\section{Experimental}

\section{Chemicals}

D,L-Cys hydrochloride monohydrate, L-Cys and D-Cys hydrochloride monohydrate were obtained from Nacalai Tesque (Kyoto, Japan), Kanto Chemical (Tokyo, Japan) and Tokyo Kasei (Tokyo, Japan), respectively. D,LHomoCys, (士)-3-mercapto-1,2-propanediol [(士)-3MPD] and ( \pm )-1-mercapto-2-propanol [(土)-1-MPR] were purchased from Nacalai Tesque, Sigma (St. Louis, MO, USA) and Aldrich (Milwaukee, WI, USA), respectively. Standard solutions of the thiols were prepared in $5 \mathrm{mM}$ acetate buffer ( $\mathrm{pH} 4.0$ ) containing $0.1 \mathrm{mM}$ EDTA $\cdot 2 \mathrm{Na}$. The chiral primary amino compounds used were L-alanine (L-Ala), L-phenylalanine ( $\mathrm{L}-$ Phe) and L-valine (L-Val); they were obtained from Takara Kosan (Tokyo, Japan). HPLC-grade methanol and acetonitrile were purchased from Kanto Chemical. The water used was purified on a Milli RO-Milli $Q$ system (Millipore, Bedford, MA, USA).

\section{HPLC system}

The HPLC system comprised a Senshu flow system 3100 pump (Senshu, Tokyo, Japan), a Rheodyne Model 7125 injector with a 20- $\mu$ l sample loop (Rheodyne, Cotati, CA, USA) and a Capcell Pak C18 AG120 column 
Table 1 Effect of L-amino acids on the retention, enantioselectivity and resolution of racemic thiols ${ }^{\mathbf{a}}$

\begin{tabular}{|c|c|c|c|c|c|c|c|c|c|}
\hline & \multicolumn{3}{|c|}{ L-Ala } & \multicolumn{3}{|c|}{ L-Phe } & \multicolumn{3}{|c|}{ L-Val } \\
\hline & $k^{\prime}$ & $\alpha$ & $R_{s}$ & $k^{\prime}$ & $\alpha$ & $R_{\mathrm{s}}$ & $k^{\prime}$ & $\alpha$ & $R_{\mathrm{s}}$ \\
\hline D,L-Cys & 0.88 & 1.00 & 0 & $\begin{array}{l}5.78 \\
7.18\end{array}$ & 1.24 & 2.82 & $\begin{array}{l}3.59 \\
4.94\end{array}$ & 1.38 & 3.44 \\
\hline D,L-HomoCys & 2.10 & 1.00 & 0 & $\begin{array}{l}11.69 \\
12.37\end{array}$ & 1.06 & 0.94 & $\begin{array}{l}7.91 \\
8.52\end{array}$ & 1.08 & 1.13 \\
\hline$( \pm)-3-M P D$ & $\begin{array}{l}1.93 \\
2.38\end{array}$ & 1.23 & 1.89 & $\begin{array}{l}11.40 \\
12.30\end{array}$ & 1.08 & 1.37 & $\begin{array}{r}8.03 \\
11.60\end{array}$ & 1.44 & 5.81 \\
\hline$( \pm)-1-M P R$ & $\begin{array}{l}4.67 \\
7.47\end{array}$ & 1.60 & 7.09 & $\begin{array}{l}26.18 \\
36.35\end{array}$ & 1.39 & 6.71 & $\begin{array}{l}16.10 \\
30.93\end{array}$ & 1.92 & 11.89 \\
\hline
\end{tabular}

a. Mobile phase: methanol- $0.05 \mathrm{M}$ sodium acetate $(40: 60, \mathrm{v} / \mathrm{v})$.

( $4.6 \mathrm{~mm}$ i.d. $\times 150 \mathrm{~mm}$; particle size, $5 \mu \mathrm{m}$ ) (Shiseido, Tokyo, Japan) with a LiChrosorb RP-18 guard column (4 mm i.d. $\times 4 \mathrm{~mm}$ ) (E. Merck, Darmstadt, Germany). The column was operated at room temperature and eluted by a mixture of $50 \mathrm{mM}$ sodium acetate and methanol or acetonitrile. The flow rate was $1.0 \mathrm{ml} / \mathrm{min}$. Detection was carried out with a Shimadzu RF-535 fluorescence HPLC monitor equipped with a flow-cell $(12 \mu \mathrm{l})$ and a xenon lamp (Ex $345 \mathrm{~nm}, \mathrm{Em} 440 \mathrm{~nm}$ ).

\section{Derivatization}

To $100 \mu \mathrm{l}$ of a thiol solution in a $1.5-\mathrm{ml}$ test tube with a glass stopper were added $100 \mu \mathrm{l}$ each of $1 \mathrm{mM}$ amino acids in water and $1 \mathrm{mM}$ OPA in a borate-phosphate buffer (pH 9.0), and then thoroughly vortexed. After allowing the mixture to stand at room temperature for about $1 \mathrm{~min}$, a $20-\mu \mathrm{l}$ aliquot of the reaction mixture was injected into the HPLC system.

\section{Results and Discussion}

The resolution of standard thiol enantiomers derivatized with OPA and $\alpha$-amino acids (L-Ala, L-Phe and LVal) was attempted on a reversed-phase column using a mobile phase of methanol- $-0.05 \mathrm{M}$ sodium acetate $(40: 60, v / v)$. Table 1 summarizes the influence of the amino acids on the capacity factor $\left(k^{\prime}\right)$, enantioseparation factor $(\alpha)$ and resolution $\left(R_{s}\right)$ of thiols. Among the $\mathrm{L}$-amino acids, L-Val gave the best selectivity for all of the thiols tested, probably owing to the presence of a bulky isopropyl group on the valyl side-chain, which gives rigidity to the isoindole diastereomers. The enantioseparation of chiral HomoCys was lowest among the thiols, probably due to the longer distance between the chiral center and the SH group in the molecule, compared with other thiols, since the optical resolution was known to depend on the proximity of the two chiral centers of the diastereomers. $^{2}$

The effect of the organic modifier in the mobile phase on the simultaneous resolution and separation of thiol enantiomers was examined. A model mixture containing D,L-Cys $(10 \mu \mathrm{M})$, D,L-HomoCys $(10 \mu \mathrm{M}),( \pm)-3-$ MPD $(2 \mu \mathrm{M})$ and $( \pm)$-1-MPR $(2 \mu \mathrm{M})$ was derivatized (a)

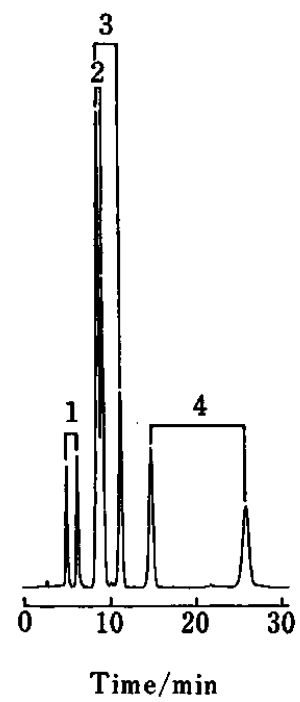

(b)

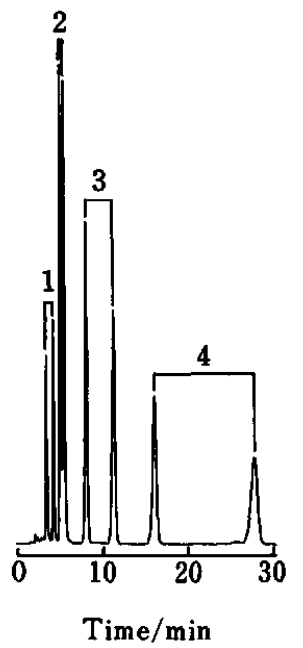

Fig. 2 Separation of thiol enantiomers by HPLC after precolumn derivatization with OPA and $\mathrm{L}-\mathrm{Val}$. Peaks: 1, D,L-Cys; 2, D,L-HomoCys; 3, (士)-3-MPD; 4, (士)-1-MPR. Mobile phase: (a), methanol- $0.05 \mathrm{M}$ sodium acetate $(45: 55$, $\mathrm{v} / \mathrm{v})$; (b), acetonitrile- $0.05 \mathrm{M}$ sodium acetate $(20: 80, \mathrm{v} / \mathrm{v})$. For other conditions see Experimental.

with OPA and L-Val. Chromatograms obtained with two eluents are shown in Fig. 2. When methanol was used as the organic modifier (Fig. 2a), a mutual separation of HomoCys and 3-MPD was not achieved, whereas acetonitrile permitted a simultaneous separation and resolution of the thiol enantiomers (Fig. 2b). The relative peak areas of Cys, HomoCys, 3-MPD and 1MPR were $100,308,1360$ and 931 , respectively. The detection limit of Cys was approximately a few picomoles, the sensitivity being much higher than that of the GITC method. ${ }^{3}$

When OPA and L-Val were used as the chiral derivatization reagents, the $D-C y s$ derivative eluted faster than the L-Cys derivative. As expected, the opposite results were obtained by the use of $\mathrm{D}-\mathrm{Val}$ instead of $\mathrm{L}-\mathrm{Val}$. In both experiments and those with higher concentrations $(100 \mu \mathrm{M})$ of $\mathrm{D}-$ and $\mathrm{L}-\mathrm{Cys}$, no racemization was 
observed during the derivatization procedure and chromatographic separation.

The proposed method seems to be useful for the HPLC analysis of trace amounts of thiol enantiomers, owing to its high sensitivity and simplicity. Quite recently, it has also been clarified that OPA reaction products formed from $N$-acetyl-D,L-cysteine and L-Val could be resolved by capillary electrophoresis. ${ }^{8}$

This paper was presented in a preliminary form at the Separation Sciences '94, Tokyo, February 22 - 23, 1994.

\section{References}

1. N. S. Srinivas and L. N. Igwemezie, Biomed. Chromatogr.,
6, 163 (1992).

2. S. Görög and M. Gazdag, J. Chromatogr. B, 659, 51 (1994).

3. S. Ito, A. Ota, K. Yamamoto and Y. Kawashima, $J$. Chromatogr., 626, 187 (1992).

4. R. H. Buck and K. Krummen, J. Chromatogr., 315, 279 (1984).

5. N. Nimura and T. Kinoshita, J. Chromatogr., 352, 169 (1986).

6. H. Nakamura and Z. Tamura, Anal. Chem., 53, 2190 (1981).

7. K. Mopper and D. Delmas, Anal. Chem., 56, 2557 (1984).

8. C. Dotte and H. Wätzig, Electrophoresis, 15, 763 (1994).

(Received December 26, 1994)

(Accepted January 27, 1995) 